Material and methods We evaluated 6 patients with osteonecrosis as a complication of leukaemia or lymphoma treatment between September 1998 and September 2013. Osteonecrosis was confirmed by magnetic resonance imaging (MRI) of the symptomatic joints.

Results Of 563 patients, 6 (4 girls, 2 boys) (1,1\%) developed symptomatic osteonecrosis, in a total of 11 joints. The median age at diagnosis of malignancy was 14 years (range 10-18 years) and the median interval between primary diagnosis and onset of osteonecrosis related symptoms was 33 months (range 11-120 months). Underlying malignancies were acute lymphoblastic leukaemia $(\mathrm{n}=3)$ and Hodgkin Lymphoma $(\mathrm{n}=3)$. Affected joints were hip $(n=7)$, knee $(n=3)$ and elbow $(n=1)$. All patients had received previous corticosteroid therapy at a median dose in prednisone equivalent of $4239 \mathrm{mg} / \mathrm{m}^{2}$ (range 3918-4600 mg/ $\mathrm{m}^{2}$ ). Treatment of osteonecrosis included restriction of weightbearing, physiotherapy and analgesics. One patient had to undergo arthrotomy. All patients showed improvement in pain and motor function.

Conclusions In our cohort, there has been a predominance of female adolescents. Weight-bearing joints were the most commonly affected. Increased awareness for skeletal symptoms during follow-up of patients with hematologic malignancies allows early detection of osteonecrosis, leading to prompt intervention and may prevent more severe morbidity.

\section{P0-0155 EVALUATION OF GROWTH HORMONE DEFICIENCY (GHD) IN CHILDREN WITH ACUTE LYMPHOBLASTIC LEUKEMIA (ALL) AND NON-HODGKIN'S LYMPHOMA (NHL)}

'S Elmi, ${ }^{2}$ REZAErfani Sayyar, ${ }^{3}$ SAM Elmi. ${ }^{1}$ Pediatric Department, Mashad University of Medical Science, Mashhad, Iran; ${ }^{2}$ Intensive Care Unit Department, Mashad University of Medical Science, Mashhad, Iran; ${ }^{3}$ Health Care Department, Mashad University of Medical Science, Mashhad, Iran

\subsection{6/archdischild-2014-307384.817}

Introduction Acute lymphoblastic leukaemia (ALL) is the most common childhood cancer and non-Hodgkin's lymphoma (NHL) is the most common childhood cancer.these children may suffer from some late effects of treatments such as endocrinopathies like thyroid, pituitary, metabolism disfunctions. Growth Hormone Deficiency (GHD) is one of the causes of short stature.

Material and methods During a 3 years cross-sectional research in Dr Sheikh children hospital in Mashhad, we studied on 50 children with ALL $(\mathrm{n}=25)$, NHL $(\mathrm{n}=25)$ who received chemotherapy alone $(n=25)$ or plus radiation $(n=25)$. Patients with height less than 5 th percentile, were evaluated for GHD via insulin stimulating test. Also for short stature workup in all patients thyroid function test were done.

Results We found 6 (12\%) children with height less than 5th percentile who $5(83.33 \%$ of them or $10 \%$ of total) had GHD. There was no statistical correlation between type of disease also type of treatment and GHD (respectively p-value $=0.667$, 0.189 ) due to small sample size. Among these 6 patients, 5 children $(83.33 \%$ of them) undergone radiation and 4 patients (66.66\% of them) had ALL. There was 2(4\% total) cases of subclinical hypothyroidism but with normal height. According to BMI (body mass index) access $2(4 \%$ total) patients had overweight.

Conclusion In our study ALL patients who received radiation had unfavourable state for height and endocrine side effects. So it seems more attention to radiotherapy complications in children with cancer especially ALLs is needed to improve their quality of life.

\section{PO-0156 POST TREATMENT THYROID DISFUNCTION AND OBESITY IN CHILDREN WITH ACUTE LYMPHOBLASTIC LEUKEMIA (ALL), NON HODGKIN'S LYMPHOMA}

'S Elmi, ${ }^{2}$ REZAErfani Sayyar, ${ }^{3}$ SAM Elmi. ${ }^{1}$ Pediatric Department, Mashad University of Medical Science, Mashhad, Iran; ${ }^{2}$ Intensive Care Unit Department, Mashad University of Medical Science, Mashhad, Iran; ${ }^{3}$ Health Care Department, Mashad University of Medical Science, Mashhad, Iran

\subsection{6/archdischild-2014-307384.818}

Background In most children with Acute Lymphoblastic Leukaemia (ALL) and Non Hodgkin's Lymphoma (NHL) who have been undergone chemotherapy with and without radiotherapy, some late effects due to treatment may occur such as endocrinopathies.

Methods We evaluated growth criteria (including short stature, obesity) and thyroid test function in 50 children with ALL ( $\mathrm{n}=$ $25)$ and NHL $(n=25)$ 3-17 year-old in remission period who randomly received chemotherapy with $(\mathrm{n}=25)$ or without $(\mathrm{n}=$ 25) radiation such as our treatment groups. The values for height, weight and BMI in less than 5th or more than 95 th percentile considers abnormal.

Results Six (12\%) patients were in less than 5th percentile height (short stature). Two patients $(4.0 \%)$ had over-weight and $48(96 \%)$ were in normal range of BMI. Six (12\%) patients were in less than 5 th and $3(6 \%)$ were in more than 95 th weight percentile. There was no significant difference between two different treatment groups for TSH $(\mathrm{p}=0.662$ (but there was a significant difference between these groups in case of $\mathrm{T} 4(\mathrm{p}=$ 0.049). Mean and SD for T4 in patients with chemotherapy alone was less than in whom received chemotherapy plus radiotherapy. There was no significant difference between ALL and NHL groups for TSH, T4 ( $\mathrm{p}=0.567,0.528$ respectively). Two boys with ALL without history of radiation had hypothyroidism that had based on their laboratory data.

Conclusion Regarding to effects of thyroid dysfunction on short stature and obesity in adolescent with ALL and NHL, we suggest to have more attention about growth, thyroid test to avoid late side effect of malignancy treatment.

\section{PO-0157 EVALUATION OF BONE MINERAL DENSITY IN CHILDREN WITH ACUTE LYMPHOBLASTIC LEUKEMIA (ALL) AND NON-HODGKIN'S LYMPHOMA (NHL)}

'S Elmi, ${ }^{2}$ REZA Erfani Sayyar, '3SAM Elmi. 'Pediatric Department, Mashad University of Medical Science, Mashhad, Iran; ${ }^{2}$ Anesthesiology and Intensive Care Department, Mashad University of Medical Science, Mashhad, Iran; ${ }^{3}$ Health Care Department, Mashad University of Medical Science, Mashhad, Iran

\subsection{6/archdischild-2014-307384.819}

Introduction Acute lymphoblastic leukaemia (ALL) and NonHodgkin's Lymphoma (NHL) are the most common childhood and adolescence malignancy respectively.

Due to the increasing survival of these children, today late side effects of teartment are important. Therapies such as corticosteroids, cytotoxic and radiotherapy effect on bone density and put the child at risk of osteoporosis and pathological fractures. 
Matherial and methods

This 3-year cross sectional study was performed in Dr. Sheikh Children's Hospital in Mashhad on 50 children with ALL ( $\mathrm{n}=$ $25)$ and NHL $(n=25)$. Half of them were received $(n=25)$ chemotherapy alone and half of them chemotherapy plus radiotherapy $(n=25)$. All children were in the remission phase. We assessed them by DEXA bone mineral densitometery (BMD) on the lumbar spine and femoral neck (hip). We also measured some bone biomarkers include calcium (ca), phosphorus (p), parathoromone (PTH), alkaline phosphatase (ALP) in plasma. Results by age, height, sex and Body Mass Index (BMI) were adjusted with a special software.

Results Mean age was $8.28 \pm 3.93$ years. There was no significant difference on bone biomarkers (Ca, P, ALP. PTH) between ALL, NHL and also between the two treatment groups. Children with ALL had lower density at the hip and lumbar spine. (respectively $\mathrm{p}$ value $<0.001$ and $\mathrm{p}$ value $=0.018$ ). A total of 50 patients, the hip BMD showed normal results in 3 patients $(6 \%)$, in 14 patients (28\%) osteopenia were seen and 33 patients $(66 \%)$ had osteoporosis. In whom received radiotherapy plus chemotherapy, one patient had normal BMD and 24 patients (48\% of total patients) at the hip and 22 patients (44\%) at lumbar spine had decreased BMD. In contrast, in whom had only chemotherapy, 24 patients (48\%) had osteoporosis at hip and 23 $(46 \%)$ at the lumbar spine. There was no significant difference in BMD between the sexes.

Conculsion Given that 94\% of children had abnormal bone density, Seem to pay more attention to the metabolic status and BMD in children with cancer can develop appropriate strategies to improve health and quality of their life.

\section{PO-0158 MANAGEMENT OF PATIENTS WITH ALL WHEN EXPOSED TO VZV}

C Glancy, R Kennedy. Paediatrics, Craigavon Area Hospital, Belfast, UK

\subsection{6/archdischild-2014-307384.820}

Aims Investigate the management of patients with ALL when exposed to Varicella Zoster Virus.

Method Retrospective study looking at all patients diagnosed with ALL between 2007-2011, a total of 60 patients. Each chart was looked at for any documented exposure to varicella and the management of the patient compared to standards set on local guidelines.

Results 58 patients in the audit. Age range was from 2 years to 15 years. 48 patients were tested at diagnosis leaving 10 patients with unknown Varicella status diagnosis.

24 patients reported exposure, 50\% of these patient exposures were significant and required treatment. 19 of these were managed appropriately and 5 were not. $100 \%$ patients that were exposed and found not to be significant exposures did not receive treatment and therefore were managed appropriately. Of the patients that were exposed and not managed according to local guidance, one patient received IVIG 2/52 after a significant exposure when according to guidance they should have received oral aciclovir. Another patient did not have their status checked at diagnosis or when exposed. The remaining three patients did not have their immune status checked at diagnosis making their management inappropriate.

Conclusion Overall the management of the patients who contacted the medical team to report exposure to Varicella were managed appropriately. Plan is to have a sticker on the front of patient notes with varicella status on diagnosis, exposure and results.

\section{P0-0159 CHILDHOOD DISSEMINATED RHABDOMYOSARCOMA OF UNKNOWN PRIMARY SITE MASQUERADING AS AN ACUTE HAEMATOLOGICAL MALIGNANCY- LITERATURE REVIEW APROPOS OF TWO UNUSUAL CASES}

${ }^{1} \mathrm{G}$ Karpinsky, ${ }^{1} \mathrm{E}$ Rak, ${ }^{2} \mathrm{M}$ Krawczyk, ${ }^{2} \mathrm{E}$ Bien. ${ }^{1}$ English Division Pediatric Oncology Scientific Circle, Medical University of Gdansk, Gdansk, Poland; '²Department of Pediatrics Hematology and Oncology, Medical University of Gdansk, Gdansk, Poland

\subsection{6/archdischild-2014-307384.821}

Background Small round cell tumours, particularly rhabdomyosarcoma (RMS), may infiltrate bone marrow (BM), mimicking acute leukaemia - both clinically and in morphological assessment of myelogram.

Aim To analyse diagnostic and therapeutic dilemmas in children with RMS masquerading as acute leukaemia.

Methods A retrospective analysis of medical charts of a 14-yearold male and 15-year-old female admitted to Department of Paediatrics, Haematology and Oncology, Medical University of Gdansk, Poland in 2007 and 2013 and literature review.

Results Both patients were referred suspected of acute leukaemia presenting with weakness, pallor, bone pains, and enlarged peripheral lymph nodes (LN). Skin bruising, petechiae and wound bleedings were progressing. Laboratory tests showed anaemia, thrombocytopenia and features of acute DIC and ATLS. BM aspiration revealed blast cells suggestive for leukaemia. Flow cytometry failed to display lymphoid or myeloid antigens. Aspirational LN biopsy revealed small round blue cells, suggesting AML, non-Hodgkin's lymphoma or Ewing's sarcoma. Modified chemotherapy in all these malignancies was introduced to alleviate DIC-associated haemorrhages and enable LN resection. Finally the diagnoses of embryonal RMS (male) and alveolar RMS (female) were made. Administration of proper chemotherapy for metastatic RMS resulted in rapid neoplasms' regression and normalisation of DIC parameters.

Conclusion Clinical presentation of childhood RMS masquerading as acute leukaemia is unique and poses diagnostic problems, especially in patients with DIC-related haemorrhages. RMS should be included in differential diagnosis of any case presenting as a systemic disease with BM infiltration of cells mimicking leukemic blasts, but lacking lymphoid and myeloid antigens in immunophenotyping by flow cytometry.

\section{P0-0160 INFECTIOUS EVENTS DURING INTENSIVE TREATMENT IN CHILDHOOD ACUTE LYMPHOBLASTIC LEUKAEMIA}

${ }^{1} \mathrm{~K}$ Martinova, ${ }^{1} \mathrm{~B}$ Coneska-Jovanova, ${ }^{1} \mathrm{~S}$ Kocheva, ${ }^{1} \mathrm{~A}$ Jovanovska, ${ }^{2} \mathrm{E}$ Bojadgieva. ${ }^{1}$ Hematology and Oncology, University Children's Hospital, Skopje, Macedonia; ${ }^{2}$ Faculty of Medical Sciences, University Goce Delcev, Stip, Macedonia

\subsection{6/archdischild-2014-307384.822}

Background Many children with acute lymphoblastic leukaemia (ALL) experience one or more infectious complications during treatment. Infections are important to study in children with ALL because they continue to contribute to morbidity and mortality, affect quality of life for children and their families and require considerable health resources to prevent and treat.

Aims To analyse the characteristics of infective episodes (I. E.) during intensive treatment (Protocol I, M and II) in children with ALL.

Methods Objective of this study ware 55 patients with ALL who were treated according to ALL-BFM 90 and ALL-BFM 95 Protocol between January 2000 and December 2007 at the University 